\title{
Signal-dependent wavelets for electromyogram classification
}

\author{
A. Maitrot · M.-F. Lucas $\cdot$ C. Doncarli $\cdot$ D. Farina
}

Published online: 10 July 2007

(C) International Federation for Medical and Biological Engineering 2007

\section{Erratum to: Med Bio Eng Comput (2005) 43(4):487-492 DOI 10.1007/BF02344730}

All results reported in the paper are valid since they have been obtained with the correct expression. The mistake in the reported expression was only due to a typing error.

The following formula:

$$
\begin{aligned}
& i=0,1: h[i]=4 \sqrt{2}\left[\left(1+(-1)^{i} \cos \alpha+\sin \alpha\right)\right. \\
& \times\left(1-(-1)^{i} \cos \beta-\sin \beta\right) \\
& \left.\left.+(-1)^{i} 2 \sin \beta \cos \alpha\right)\right] \\
& i=2,3: h[i]=2 \sqrt{2}\left[1+\cos (\alpha-\beta)+(-1)^{i} \sin (\alpha-\beta)\right] \\
& i=4,5: h[i]=1 / \sqrt{2}-h(i-4)-h(i-2)
\end{aligned}
$$

reported at page 488 , should read:

$$
\begin{aligned}
& \begin{aligned}
i=0,1: h[i]=[ & \left(1+(-1)^{i} \cos \alpha+\sin \alpha\right) \\
& \times\left(1-(-1)^{i} \cos \beta-\sin \beta\right) \\
& \left.\left.+(-1)^{i} 2 \sin \beta \cos \alpha\right)\right] /(4 \sqrt{2})
\end{aligned} \\
& \begin{aligned}
i=2,3: h[i]=\left[1+\cos (\alpha-\beta)+(-1)^{i} \sin (\alpha-\beta)\right] /(2 \sqrt{2}) \\
i=4,5: h[i]=1 / \sqrt{2}-h(i-4)-h(i-2)
\end{aligned}
\end{aligned}
$$

The online version of the original article can be found under doi:10.1007/BF02344730.

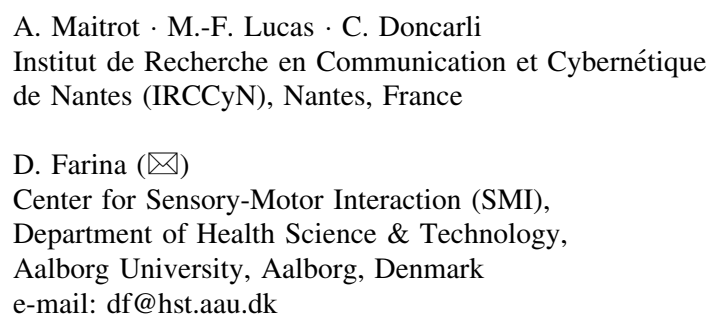

